PROCEEDINGS OF THE

AMERICAN MATHEMATICAL SOCIETY

Volume 127, Number 12, Pages 3677-3683

S 0002-9939(99)04975-8

Article electronically published on May 11, 1999

\title{
CONVERGENCE OF THE STEEPEST DESCENT METHOD FOR ACCRETIVE OPERATORS
}

\author{
CLAUDIO H. MORALES AND CHARLES E. CHIDUME
}

(Communicated by Palle E. T. Jorgensen)

\begin{abstract}
Let $X$ be a uniformly smooth Banach space and let $A: X \rightarrow X$ be a bounded demicontinuous mapping, which is also $\alpha$-strongly accretive on $X$. Let $z \in X$ and let $x_{0}$ be an arbitrary initial value in $X$. Then the approximating scheme

$$
x_{n+1}=x_{n}-c_{n}\left(A x_{n}-z\right), \quad n=0,1,2, \ldots,
$$

converges strongly to the unique solution of the equation $A x=z$, provided that the sequence $\left\{c_{n}\right\}$ fulfills suitable conditions.
\end{abstract}

\section{INTRODUCTION}

The main objective of this paper is to present some further findings concerning some recent works (see [15], [17]) in the area of the so-called steepest descent approximation method for accretive operators. First of all, we describe the general setting of our results.

Let $X$ be a (real) Banach space and let $D$ be a subset of $X$. An operator $A: D \rightarrow X$ is said to be $k$-accretive $(k \in \mathbb{R})$ if for each pair $u, v \in D$ there exists $j \in J(u-v)$ such that

$$
\langle A(u)-A(v), j\rangle \geq k\|u-v\|^{2},
$$

where $J: X \rightarrow 2^{X^{*}}$ is the normalized duality mapping which is defined by

$$
J(u)=\left\{j \in X^{*}:\langle u, j\rangle=\|u\|^{2},\|j\|=\|u\|\right\} .
$$

For an alternative equivalent definition see Morales [11]. Here $\langle\cdot, \cdot\rangle$ denotes the generalized duality pairing. It is an immediate consequence of the Hahn-Banach Theorem that $J(u)$ is nonempty for each $u \in X$. Moreover, it is known that $J(u)$ is single-valued if $X$ is smooth (in particular when the dual space $X^{*}$ is strictly convex), while if $X^{*}$ is uniformly convex, then the mapping $J$ is uniformly continuous on bounded sets. For $k>0$ in inequality (1), we say that $A$ is strongly accretive, while for $k=0, A$ is called accretive. There is a class of mappings intimately related to the $k$-accretive ones. These are the $k$-pseudo-contractions (for more details see [11]). This latter family is formed by mappings written as $I-A$ where $I$ is the identity and $A$ is $k$-accretive. Nevertheless, in the present paper,

Received by the editors October 21, 1996 and, in revised form, June 20, 1997, November 6, 1997, and February 27, 1998.

1991 Mathematics Subject Classification. Primary 47H10.

Key words and phrases. Uniformly smooth space, $\alpha$-strongly accretive.

(C)1999 American Mathematical Society 
we are mainly interested in a class of operators somehow more general than the strongly accretive ones, which are defined as follows.

Let $\alpha:[0, \infty) \rightarrow[0, \infty)$ be a function for which $\alpha(0)=0$ and the $\liminf _{r \rightarrow r_{0}} \alpha(r)$ $>0$ for every $r_{0}>0$. An operator $A: D(A) \subset X \rightarrow X$ is called $\alpha$-strongly accretive if for each $u, v \in D(A)$ there exists $j \in J(u-v)$ so that

$$
\langle A u-A v, j\rangle \geq \alpha(\|u-v\|)\|u-v\| .
$$

In contrast with the terminology of [12] we do not assume that $\alpha$ is continuous at zero, nor do we assume that $\alpha$ is strictly increasing, another common assumption. Nevertheless, a mild additional assumption will be imposed on the function $\alpha$. We also say that $T$ is $\alpha$-strongly pseudo-contractive if $I-T$ is $\alpha$-strongly accretive.

Along with existence theory for zeros of monotone and accretive operators, we find through the years significant efforts to identify approximation schemes for such zeros. Particularly the so-called steepest descent method was introduced for monotone operators by Vainberg [16] and Zarantonello [18] independently, in the following terms.

Let $A$ be a monotone and Lipschitz operator defined on a Hilbert space into itself. Then the approximating process

$$
x_{n+1}=(1-\lambda) x_{n}-\lambda A x_{n}, \quad \lambda \in(0,1),
$$

converges strongly to a zero of $A$.

In the last 30 years the process described by (2) has evolved in various directions. Among them, we can find Petryshyn [13], Bruck [1], Crandall and Pazy [5], Dotson [7] and many others. Nowadays, this process is described for accretive operators as follows:

$$
x_{n+1}=x_{n}-c_{n} A x_{n}
$$

for a suitable sequence $\left\{c_{n}\right\}$.

Recently Xu and Roach [17], and Chidume [4] have studied necessary and sufficient conditions for the convergence of the process (3) for these operators. The purpose of this paper is to continue this study. Among other things, we repair what seems to be an oversight in the proof of Theorem 1 of [17], which is also repeated in [4]. Specifically, in trying to show the boundedness of the sequence $\left\{x_{n}\right\}$ on page 347 of [17], the authors establish formula (2.11) for $n=n_{j}$, but not necessarily for its successor. The step seems crucial and is referred to later in the argument. In what follows, we give an alternative proof of boundedness of this sequence which appears shorter and simpler, although our proof is somewhat inspired by the original ideas. At the same time we also extend some results of [3] and [6].

We will need a few preliminary facts, in particular, the following lemma.

Lemma 1 (Reich [14]). Let $X$ be a uniformly smooth Banach space. Then there exists a nondecreasing continuous function $b:[0, \infty) \rightarrow[0, \infty)$ which satisfies the following:

(i) $b(c t) \leq c b(t)$ for all $c \geq 1$;

(ii) $\lim _{t \rightarrow 0^{+}} b(t)=0$; and

(iii) $\|x+y\|^{2} \leq\|x\|^{2}+2 \operatorname{Re}\langle y, J(x)\rangle+\max \{\|x\|, 1\}\|y\| b(\|y\|)$ for all $x, y \in X$.

Throughout the paper we assume that $X$ is a real Banach space whose dual space $X^{*}$ is uniformly convex. This latter statement is equivalent to saying that $X$ is uniformly smooth. In addition, we say that an operator $A$ is demicontinuous if it is continuous from the strong topology of $X$ into the weak topology of $X$. We 
also say that $A$ is bounded if it maps bounded sets into bounded sets. We denote the distance between the sets $A$ and $B$ by $\operatorname{dist}(A, B)$, where

$$
\operatorname{dist}(A, B)=\inf \{\|a-b\|: a \in A, b \in B\} .
$$

\section{MAin RESUlts}

Theorem 2. Let $X$ be a uniformly smooth Banach space, let $b$ be the function of Lemma 1, and let $A: X \rightarrow X$ be a bounded demicontinuous mapping, which is also $\alpha$-strongly accretive on $X$. Let $z \in X$ and let $x_{0}$ be an arbitrary initial value in $X$ for which the $\liminf _{r \rightarrow \infty} \alpha(r)>\left\|A\left(x_{0}\right)\right\|$. Then the approximating scheme

$$
x_{n+1}=x_{n}-c_{n}\left(A x_{n}-z\right), \quad n=0,1,2, \ldots,
$$

converges strongly to the unique solution of the equation $A x=z$, provided that the sequence $\left\{c_{n}\right\}$ of positive real numbers satisfies the following:

(i) $\left\{c_{n}\right\}$ is bounded above by the constant $r_{0}$ defined in (6);

(ii) $\sum_{n=1}^{\infty} c_{n}=\infty$; and

(iii) $\sum_{n=0}^{\infty} c_{n} b\left(c_{n}\right)<\infty$.

We begin with some preliminary notions and notations that will be used in the proof of Theorem 2. We first observe that due to assumption (iii) on $\left\{c_{n}\right\}$, we easily derive that $\lim _{n \rightarrow \infty} c_{n}=0$.

For the initial guess $x_{0}$ of the iterative process defined by (4), we define the positive constants $m_{0}$ and $r_{0}$ as follows. We may assume without loss of generality that $z=0$ and consequently that $\left\|A x_{0}\right\|>0$. Then we set

$$
a_{0}=\sup \left\{r: \alpha(r) \leq\left\|A x_{0}\right\|\right\},
$$

which allows us to define

$$
m_{0}=\sup \left\{\|A u\|:\left\|u-x_{0}\right\| \leq 3 a_{0}\right\}
$$

and

$$
r_{0}=\frac{1}{m_{0}} \min \left\{\sup b^{-1}\left[\frac{a_{0} \alpha\left(a_{0}\right)}{\max \left\{a_{0}, 1\right\} m_{0}}\right], a_{0}\right\} .
$$

Proposition 3. Let $X$ be a Banach space, whose dual $X^{*}$ is uniformly convex. Let $A: D(A) \subset X \rightarrow X$ be a bounded and $\alpha$-strongly accretive operator. Suppose there exists a zero of $A$ and a sequence defined by

$$
x_{n+1}=x_{n}-c_{n} A x_{n}, \quad n=0,1,2, \ldots,
$$

with $c_{n} \leq r_{0}$ for $n \in \mathbb{N}$. Then $\left\{x_{n}\right\}$ is bounded.

Proof. We denote by $x^{*}$ the unique zero of $A$. Then

$$
\alpha\left(\left\|x_{0}-x^{*}\right\|\right)\left\|x_{0}-x^{*}\right\| \leq\left\langle A x_{0}, J\left(x_{0}-x^{*}\right)\right\rangle \leq\left\|A x_{0}\right\|\left\|x_{0}-x^{*}\right\| .
$$

This implies that $\alpha\left(\left\|x_{0}-x^{*}\right\|\right) \leq\left\|A x_{0}\right\|$, and thus $\left\|x_{0}-x^{*}\right\| \leq a_{0}$. Suppose now that the sequence $\left\{x_{n}\right\}$ is not bounded. So, let $n_{0}$ be the first natural number for which

$$
\left\|x_{n_{0}}-x^{*}\right\| \geq a_{0} .
$$


Then $\left\|x_{n_{0}-1}-x^{*}\right\|<a_{0}$, and thus $\left\|x_{n_{0}-1}-x_{0}\right\| \leq 2 a_{0}$. This implies that $\left\|A x_{n_{0}-1}\right\| \leq m_{0}$. Therefore,

$$
\begin{aligned}
\left\|x_{n_{0}+1}-x^{*}\right\|^{2}= & \left\|x_{n_{0}}-c_{n_{0}} A x_{n_{0}}-x^{*}\right\|^{2} \\
\leq & \left\|x_{n_{0}}-x^{*}\right\|^{2}-2 c_{n_{0}}\left\langle A x_{n_{0}} J\left(x_{n_{0}}-x^{*}\right)\right\rangle \\
& +\max \left\{\left\|x_{n_{0}}-x^{*}\right\|, 1\right\} c_{n_{0}}\left\|A x_{n_{0}}\right\| b\left(c_{n_{0}}\left\|A x_{n_{0}}\right\|\right) .
\end{aligned}
$$

However, for further estimates of inequality (8), we need to show that $\left\|A x_{n_{0}}\right\| \leq m_{0}$. To see this, let

$$
\begin{aligned}
\left\|x_{n_{0}}-x_{0}\right\| & \leq\left\|x_{n_{0}}-x_{n_{0}-1}\right\|+\left\|x_{n_{0}-1}-x^{*}\right\|+\left\|x^{*}-x_{0}\right\| \\
& \leq c_{n_{0}-1}\left\|A x_{n_{0}-1}\right\|+2 a_{0} \leq r_{0} m_{0}+2 a_{0} \leq 3 a_{0},
\end{aligned}
$$

which means $\left\|A x_{n_{0}}\right\| \leq m_{0}$. Now if we denote $k_{0}=\max \left\{\left\|x_{n_{0}}-x^{*}\right\|, 1\right\}$, then we may re-write the inequality (8) as

$$
\begin{aligned}
\left\|x_{n_{0}+1}-x^{*}\right\|^{2} \leq & \left\|x_{n_{0}}-x^{*}\right\|^{2}-2 c_{n_{0}} \alpha\left(\left\|x_{n_{0}}-x^{*}\right\|\right)\left\|x_{n_{0}}-x^{*}\right\| \\
& +k_{0} c_{n_{0}} m_{0} b\left(c_{n_{0}} m_{0}\right) \\
\leq & \left\|x_{n_{0}}-x^{*}\right\|^{2}-c_{n_{0}}\left[2 a_{0} \alpha\left(a_{0}\right)-k_{0} m_{0} b\left(c_{n_{0}} m_{0}\right)\right] .
\end{aligned}
$$

However, due to the choice of $r_{0}$ in (6), it is easy to see that

$$
2 a_{0} \alpha\left(a_{0}\right)-k_{0} m_{0} b\left(c_{n_{0}} m_{0}\right) \geq 0 .
$$

Therefore

$$
\left\|x_{n_{0}+1}-x^{*}\right\| \leq\left\|x_{n_{0}}-x^{*}\right\| .
$$

To complete the proof, we first defined $\rho_{n}$ by $\left\|x_{n}-x^{*}\right\|$. Then we have that $\rho_{n_{0}+1} \leq \rho_{n_{0}}$. In addition, it can be easily derived that

$$
\left\|A x_{n_{0}+1}\right\| \leq m_{0} \text {. }
$$

This means, if we assume that $\rho_{n_{0}+1} \geq a_{0}$, then the previous argument holds and thus $\rho_{n_{0}+2} \leq \rho_{n_{0}+1}$. On the other hand, if $\rho_{n_{0}+1}<a_{0}$, then either $\rho_{n}<a_{0}$ for all $n \geq n_{0}+1$, in which case the proof is complete, or there exists $j \in \mathbb{N}$ such that $\rho_{j} \geq a_{0}$ while $\rho_{j-1}<a_{0}$. In the latter case, if $\left\|A x_{j-1}\right\| \leq m_{0}$ we can return to the previous argument given in (7)-(9). To this end, we note that

$$
\begin{aligned}
\left\|x_{j}-x^{*}\right\| & \leq\left\|x_{j}-x_{j-1}\right\|+\left\|x_{j-1}-x^{*}\right\| \\
& \leq c_{j-1}\left\|A x_{j-1}\right\|+a_{0},
\end{aligned}
$$

while,

$$
\left\|x_{j-1}-x_{0}\right\| \leq\left\|x_{j-1}-x^{*}\right\|+\left\|x^{*}-x_{0}\right\| \leq 2 a_{0} .
$$

This implies that $\left\|A x_{j-1}\right\| \leq m_{0}$, and therefore $\left\|x_{n}-x^{*}\right\| \leq 2 a_{0}$ for all $n \in \mathbb{N}$, proving that the sequence $\left\{x_{n}\right\}$ is bounded.

Proof of Theorem 2. Due to Theorem 1 of Kartsatos [8], we conclude that $A(X)$ is open in $X$. Since $A(X)$ is also clearly closed, $A$ must be surjective. This means, the equation $A x=z$ has a unique solution for an arbitrary $z \in X$ and without loss of generality we may assume that $z=0$.

Due to Proposition 3 we know that $\left\{x_{n}\right\}$ is bounded and thus so is $\left\{A x_{n}\right\}$. Therefore, this and (9) imply

$$
\left\|x_{n+1}-x^{*}\right\|^{2} \leq\left\|x_{n}-x^{*}\right\|^{2}-2 c_{n} \alpha\left(\left\|x_{n}-x^{*}\right\|\right)\left\|x_{n}-x^{*}\right\|+M c_{n} b\left(c_{n}\right)
$$


for a suitable positive constant $M$. It follows recursively that for $n>1$

$$
\left\|x_{n+1}-x^{*}\right\|^{2} \leq\left\|x_{1}-x^{*}\right\|^{2}-2 \sum_{j=1}^{n} c_{j} \alpha\left(\left\|x_{j}-x^{*}\right\|\right)\left\|x_{j}-x^{*}\right\|+M \sum_{j=1}^{n} c_{j} b\left(c_{j}\right) .
$$

Now, using condition (ii) of $\left\{c_{n}\right\}$ we obtain

$$
\sum_{j=1}^{\infty} c_{j} \alpha\left(\left\|x_{j}-x^{*}\right\|\right)\left\|x_{j}-x^{*}\right\|<\infty
$$

and since $\sum_{j=1}^{\infty} c_{j}=+\infty$, we conclude that

$$
\liminf _{n \rightarrow \infty} \alpha\left(\left\|x_{n}-x^{*}\right\|\right)\left\|x_{n}-x^{*}\right\|=0 .
$$

Consequently, there exists a subsequence $\left\{x_{n_{k}}\right\}$ of $\left\{x_{n}\right\}$ for which

$$
\lim _{k \rightarrow \infty}\left\|x_{n_{k}}-x^{*}\right\|=0
$$

To complete the proof, let $\varepsilon>0$ be arbitrary. Then there exists a $k_{0} \in \mathbb{N}$ such that

$$
\left\|x_{k_{0}}-x^{*}\right\|<\frac{\varepsilon}{\sqrt{2}} \text { and } \sum_{j=k_{0}}^{\infty} c_{j} b\left(c_{j}\right)<\frac{\varepsilon^{2}}{2 M} .
$$

Now we let $n \geq k_{0}$ and apply a recursive process to (10). Then

$$
\begin{aligned}
\left\|x_{n+1}-x^{*}\right\|^{2} & \leq\left\|x_{k_{0}}-x^{*}\right\|^{2}-2 \sum_{j=k_{0}}^{n} \alpha\left(\left\|x_{j}-x^{*}\right\|\right)\left\|x_{j}-x^{*}\right\|+M \sum_{j=k_{0}}^{n} c_{j} b\left(c_{j}\right) \\
& \leq\left\|x_{k_{0}}-x^{*}\right\|^{2}+M \sum_{j=k_{0}}^{\infty} c_{j} b\left(c_{j}\right)<\frac{\varepsilon^{2}}{2}+\frac{\varepsilon^{2}}{2}=\varepsilon^{2} .
\end{aligned}
$$

Therefore the sequence $\left\{x_{n}\right\}$ converges strongly to the unique zero of the operator $A-z$.

The study of convergence of the Mann process for operators defined on closed and convex subsets of a Banach space into itself has been of interest in recent years (see for instance [2], [9], [15]). Our next result deals precisely with the case that the operator $A$ is not necessarily defined in the whole Banach space $X$.

Before we state our next theorem, we need the following existence result for zeros of an operator, which also appears to be a new result.

Proposition 4. Let $K$ be a closed convex subset of a Banach space $X$, let $A: K \rightarrow$ $X$ be a continuous and $\alpha$-strongly accretive mapping, where the $\liminf _{r \rightarrow \infty} \alpha(r)>$ $\left\|A\left(x_{0}\right)\right\|$ for some $x_{0} \in K$. Suppose in addition that

$$
\lim _{h \rightarrow 0} \frac{1}{h} \operatorname{dist}(x-h A x ; K)=0
$$

for every $x \in K$. Then $A$ has a unique zero in $K$.

Proof. Without loss of generality we may assume $x_{0}=0$. Since the operator $-A$ fulfills the assumptions of Theorem 6 of Martin [10], we conclude that $K \subset$ $(I+A)(K)$. Also the fact that $I+A$ is invertible implies that $g=(I+A)^{-1}$ is a non-expansive mapping from $K$ into $K$. Since the fixed points of $g$ are the zeros of $A$, it is sufficient to show that $g$ has a fixed point in $K$. To this end, we first show that the set

$$
E=\{x \in K: A x=t x \text { for some } t<0\}
$$


is bounded. To see this, let $x \in E$. Then $A x=t x$ for some $t<0$ and,

$$
\langle A x-A(0), j\rangle \geq \alpha(\|x\|)\|x\|,
$$

for some $j \in J(x)$. This implies,

$$
\alpha(\|x\|)\|x\| \leq t\|x\|^{2}+\|A(0)\|\|x\| .
$$

Since $t<0, \alpha(\|x\|) \leq\|A(0)\|$, and this implies that $E$ is bounded. As a consequence of this we can easily show that the set

$$
F=\{y \in K: g(y)=\lambda y \text { for some } \lambda>1\}
$$

is also bounded. Next, we show that $(I-g)(K)$ is a closed set of $X$. Suppose $\left\{y_{n}\right\}$ is a sequence in $K$ so that $y_{n}-g\left(y_{n}\right) \rightarrow u$, for some $u \in X$. Let $g\left(y_{n}\right)=x_{n}$. Then $y_{n}-g\left(y_{n}\right)=A x_{n} \rightarrow u$. But

$$
\left\langle A x_{n}-A x_{m}, j\right\rangle \geq \alpha\left(\left\|x_{n}-x_{m}\right\|\right)\left\|x_{n}-x_{m}\right\|,
$$

which implies that $\alpha\left(\left\|x_{n}-x_{m}\right\|\right) \leq\left\|A x_{n}-A x_{m}\right\|$, and thus $\left\{x_{n}\right\}$ is a Cauchy sequence. This means $x_{n} \rightarrow x$ for some $x \in K$, and since $I+A$ is continuous, $y_{n} \rightarrow y$ for some $y \in K$. This implies $u=(I-g)(y)$.

Now, let $t_{n} \in(0,1)$ so that $t_{n} \rightarrow 1^{-}$as $n \rightarrow \infty$. Then $t_{n} g\left(y_{n}\right)=y_{n}$ for some $y_{n} \in K$, which implies that

$$
y_{n}-g\left(y_{n}\right)=\left(1-t_{n}^{-1}\right) y_{n} .
$$

Since $\left\{y_{n}\right\} \subset F, y_{n}-g\left(y_{n}\right) \rightarrow 0 \in(I-g)(K)$.

Proposition 4 is an extension of Theorem 2 of Deimling [6], who assumes in addition that either there exists a number $R>0$ and $j \in J(x)$ so that $\langle A x, j\rangle \geq 0$ for $\|x\| \geq R$ or $\|A x\| \rightarrow \infty$ as $\|x\| \rightarrow \infty$.

Corollary 5. Let $X$ and $K$ be as in Proposition 4. Let $T: K \rightarrow K$ be a continuous and $\alpha$-strongly pseudo-contractive mapping. Then $T$ has a unique fixed point in $K$.

We conclude with a result that improves Theorem 1 of [3].

Theorem 6. Let $X$ be a uniformly smooth Banach space and let $K$ be a closed convex subset of $X$. Suppose $A: K \rightarrow X$ is a bounded, continuous and $\alpha$-strongly accretive operator for which $I-A$ maps $K$ into $K$. Then the iterative process (3) converges strongly to the unique zero of $A$ provided that $\left\{c_{n}\right\}$ satisfies the same assumptions as in Theorem 2.

Proof. Due to Corollary 5, the operator $A$ has a unique zero in $K$, and since continuity implies demicontinuity, the conclusion follows from Theorem 2.

\section{ACKNOWLEDGMEnT}

The authors wish to thank the referees for many valuable suggestions to improve the writing of this manuscript.

\section{REFERENCES}

1. R. E. Bruck, Jr., The iterative solution of the equation $y \in x+T x$ for a monotone operator $T$ in Hilbert space, Bull. Amer. Math. Soc. 79 (1973), 1258-1261. MR 48:7034

2. C. E. Chidume, Iterative approximation of fixed points of Lipschitzian strictly pseudocontractive mappings, Proc. Amer. Math. Soc. 99 (1987), 283-288. MR 87m:47122

3. Math. Soc. 120 (1994), 545-551. MR 94d:47056 
4. - Steepest descent approximations for accretive operator equations, Nonlinear Anal. Theory Methods Appl. 26 (1996), 299-311. MR 97b:47067

5. M. G. Crandall and A. Pazy, On the range of accretive operators, Israel J. Math. 27 (1977), 235-246. MR 56:1142

6. K. Deimling, Zeros of accretive operators, Manuscripta Math. 13 (1974), 365-374. MR 50:3030

7. W. G. Dotson, Jr., An iterative process for monotonic nonexpansive operators in Hilbert Space, Math. Comp. 32 (1978), 223-225. MR 57:10524

8. A. G. Kartsatos, Zeros of demicontinuous accretive operators in Banach spaces, J. Integral Eqns. 8 (1985), 175-184. MR 86j:47078

9. L. Liu, Ishikawa and Mann iterative process with errors for nonlinear strongly accretive mappings in Banach spaces, J. Math. Anal. Appl. 194 (1995), 114-125. MR 97g:47069

10. R. H. Martin, Differential equations on closed subsets of a Banach space, Trans. Amer. Math. Soc. 179 (1973), 399-414. MR 47:7537

11. C. H. Morales, Zeros for accretive operators satisfying certain boundary conditions, J. Math. Anal. Appl. 105 (1985), 167-175. MR 86h:47090

12. - Surjectivity theorems for multi-valued mappings of accretive type, Comment. Math. Univ. Carol. 26 (1985), 397-413. MR 87c:47074

13. W. V. Petryshyn, On the extension and solution of nonlinear operator equations, Illinois J. Math. 10 (1966), 255-274. MR 34:8242

14. S. Reich, An iterative procedure for constructing zeros of accretive sets in Banach spaces, Nonlinear Anal. Theory Methods Appl. 2 (1978), 85-92. MR 81b:47065

15. K. K. Tan and H. K. Xu, Iterative solutions to nonlinear equations of strongly accretive operators in Banach spaces, J. Math. Anal. Appl. 178 (1993), 9-21. MR 94g:47085

16. M. M. Vainberg, On the convergence of the method of steepest descent for nonlinear equations, Sibirsk Mat. Z. (1961), 201-220. MR 23A:4026

17. Z. Xu and G. F. Roach, A necessary and sufficient condition for convergence of steepest descent approximation to accretive operator equations, J. Math. Anal. Appl. 167 (1992), 340-354. MR 93e: 47086

18. E. H. Zarantonello, The closure of the numerical range contains the spectrum, Bull. Amer. Math. Soc. 70 (1964), 781-783. MR 30:3389

Department of Mathematics, University of Alabama in Huntsville, Huntsville, AlABAMA 35899

E-mail address: morales@math.uah.edu

International Centre for Theoretical Physics, P. O. Box 586, 34100, Trieste, Italy

E-mail address: chidume@ictp.trieste.it 